\title{
Restrictions of characters in $p$-solvable groups
}

\author{
Damiano Rossi* and Benjamin Sambale ${ }^{\dagger}$
}

July 23, 2021

\begin{abstract}
Let $G$ be a $p$-solvable group, $P \leq G$ a $p$-subgroup and $\chi \in \operatorname{Irr}(G)$ such that $\chi(1)_{p} \geq|G: P|_{p}$. We prove that the restriction $\chi_{P}$ is a sum of characters induced from subgroups $Q \leq P$ such that $\chi(1)_{p}=|G: Q|_{p}$. This generalizes previous results by Giannelli-Navarro and Giannelli-Sambale on the number of linear constituents of $\chi_{P}$. Although this statement does not hold for arbitrary groups, we conjecture a weaker version which can be seen as an extension of Brauer-Nesbitt's theorem on characters of $p$-defect zero. It also extends a conjecture of Wilde.
\end{abstract}

Keywords: $p$-solvable groups; character restriction; linear constituents

AMS classification: 20C15, 20D20

\section{Introduction}

Let $p$ be a prime and let $G$ be a finite group whose order is divisible by $p^{n}$, but not by $p^{n+1}$. Recall that an irreducible character $\chi \in \operatorname{Irr}(G)$ has $p$-defect 0 , if the degree $\chi(1)$ is divisible by $p^{n}$. A well-known theorem by Brauer and Nesbitt [3, Theorem 1] asserts that $\chi$ vanishes on all elements $g \in G$ of order divisible by $p$. Equivalently, the restriction of $\chi$ to a Sylow $p$-subgroup $P$ of $G$ is a multiple of the regular character of $P$. In particular, all irreducible characters $\theta \in \operatorname{Irr}(P)$ appear as constituents of $\chi_{P}$ with multiplicity at least $\theta(1)$ in this case. In [7, 8, 9], Giannelli and Navarro investigated a more general situation where $\chi(1)$ is divisible by $p$ and $\chi_{P}$ has at least one linear constituent $\lambda \in \operatorname{Irr}(P)$. They conjectured (and proved in many cases) that $\chi_{P}$ has at least $p$ distinct linear constituents. For $p$-solvable groups $G$, they actually showed the stronger statement that $\left(\lambda_{Q}\right)^{P}$ is a summand of $\chi_{P}$ for some subgroup $Q \leq P$ with index $|P: Q|=p$.

In a subsequent paper [10, Giannelli and the second author studied the following blockwise version of the conjecture. Let $B$ be a $p$-block of $G$ with defect group $D$. Let $\chi \in \operatorname{Irr}(B)$ be of positive height and assume that $\chi_{D}$ has a linear constituent. Then $\chi_{D}$ has at least $p$ distinct linear constituents. Although we proved this conjecture in some cases, the $p$-solvable group case was left open at that time.

Meanwhile we realized that the restriction to Sylow subgroups or to defect groups was unnecessary and perhaps misleading in the latter case. The aim of the present paper is to prove the following more general theorem and its corollary for $p$-solvable groups.

\footnotetext{
*Arbeitsgruppe Algebra und Zahlentheorie, Bergische Universität Wuppertal, Gaußstraße 20, 42119 Wuppertal, Germany, rossi@uni-wuppertal.de

${ }^{\dagger}$ Institut für Algebra, Zahlentheorie und Diskrete Mathematik, Leibniz Universität Hannover, Welfengarten 1, 30167 Hannover, Germany, sambale@math.uni-hannover.de
} 
Theorem 1. Let $P$ be a Sylow p-subgroup of a finite $p$-solvable group $G$. If $\chi \in \operatorname{Irr}(G)$, then $\chi_{P}$ is a sum of characters induced from subgroups $Q \leq P$ such that $\chi(1)_{p}=|P: Q|$.

The statement of Theorem 1] was of course inspired by [9, Theorems B and C]. By using the Mackey formula, we can extend the above result to arbitrary $p$-subgroups.

Corollary 2. Let $P$ be a p-subgroup of a finite $p$-solvable group $G$. Let $\chi \in \operatorname{Irr}(G)$ such that $\chi(1)_{p} \geq$ $|G: P|_{p}$. Then $\chi_{P}$ is a sum of characters induced from subgroups $Q \leq P$ such that $\chi(1)_{p}=|G: Q|_{p}$.

In fact, under certain circumstances, $p$-solvable groups can be replaced by $\pi$-separable groups where $\pi$ is a set of primes. The details are outlined after the proof of Theorem 1 below. If $G=P$, then Theorem 1 is the well-known fact that characters of $p$-groups are monomial.

The following consequence of Theorem 1 was originally proven by Navarro (private communication).

Corollary 3. Let $P$ be a Sylow p-subgroup of a p-solvable group $G$. Then for every $\chi \in \operatorname{Irr}(G)$ there exist $Q \leq P$ and a linear character $\lambda \in \operatorname{Irr}(Q)$ such that $\chi(1)_{p}=|P: Q|$ and $\lambda^{P}$ is a summand of $\chi_{P}$.

Using Kessar-Malle's theorem [14] on the height zero conjecture, Giannelli-Navarro [9, Theorem C] have shown that Corollary 3 remains true for arbitrary groups $G$ whenever $P$ is abelian (here $Q$ can be chosen as a defect group of the block containing $\chi$ ). The next corollary settles the open question in [10] mentioned above.

Corollary 4. Let $B$ be a p-block of a p-solvable group $G$ with defect group $D$. Let $\chi \in \operatorname{Irr}(B)$ be of positive height such that $\chi_{D}$ has a linear constituent. Then $\chi_{D}$ has at least $p$ distinct linear constituents.

Initially, we believed that the statement of Theorem 1 could hold for arbitrary groups, until we found the counterexample PSU $(5,2)$ for $p=2$. Nevertheless, we did not find any counterexamples to the following weaker version (which is algorithmically much easier to check).

Conjecture 5. Let $P$ be a Sylow p-subgroup of a finite group $G$ and let $\chi \in \operatorname{Irr}(G)$. Then $\chi_{P}$ is an integral linear combination of characters induced from subgroups $Q \leq P$ such that $\chi(1)_{p}=|P: Q|$.

Just like Corollary 2, the conjecture implies an analogue statement for arbitrary $p$-subgroups.

Conjecture 5 relates to a strong form of Brauer's induction theorem due to Willems [21]. If $\chi$ is realized by a module with vertex $Q \leq P$, then Willems' theorem implies that $\chi_{P}$ is an integral linear combination of characters induced from subgroups of the form $P \cap Q^{g}$ where $g \in G$. In particular, if $\chi$ has height 0 , then $\left|P: P \cap Q^{g}\right| \geq|P: Q| \geq \chi(1)_{p}$. Therefore, Conjecture 5 holds whenever $P$ is abelian by Kessar-Malle [14]. In general we cannot expect to find a vertex $Q$ such that $\chi(1)_{p} \leq|P: Q|$ (see 4]).

If $P=\langle g\rangle \leq G$ is an arbitrary cyclic $p$-subgroup, then there is only one choice for $Q$. In this case Conjecture 5 implies that $\chi(g)=0$ as long as $\chi(1)_{p}>|G: P|_{p}$. This is a special case of Wilde's Conjecture [20, Conjecture 1.1] (for $p$-elements). Apart from $p$-solvable groups, Wilde proved his conjecture also for symmetric groups. If true, Conjecture 5 would also generalize the Brauer-Nesbitt theorem on characters of $p$-defect 0 .

In the next section we prove Theorem 1 and its corollaries. Then, we study minimal counterexamples to Conjecture 5. Among other results, we prove Conjecture 5 whenever $G$ is one of the simple groups $\operatorname{PSL}(2, q)$ or $\mathrm{Sz}(q)$ as well as if $P$ is a dihedral or quaternion 2-group. In these cases the integral linear combination has positive coefficients so that the statement of Theorem 1 remains true. 


\section{Proofs}

Our notation is standard and follows Huppert's book [13] and Navarro's books [15, 16]. We will make use of $\pi$-special characters where $\pi$ is a set of primes (in fact, $\pi=\{p\}$ or $\pi=p^{\prime}$ ). Although the definition of a $\pi$-special character $\chi$ is somewhat technical, the reader only needs to know that every prime divisor of $\chi(1)$ lies in $\pi$.

Lemma 6. Let $P$ be a Sylow p-subgroup of a finite group $G$. If $\chi \in \operatorname{Irr}(G)$ is imprimitive and Theorem 1 holds for every proper subgroup of $G$, then Theorem 1 holds for $\chi$.

Proof. Since $\chi$ is imprimitive, there exist $H<G$ and $\psi \in \operatorname{Irr}(H)$ such that $\chi=\psi^{G}$. We may choose $P$ such that $S:=H \cap P$ is a Sylow $p$-subgroup of $H$. Since Theorem 1 holds for $H$, we may write $\psi_{S}=\sum_{i=1}^{n} \lambda_{i}^{S}$ where $\lambda_{i} \in \operatorname{Irr}\left(Q_{i}\right)$ and $Q_{i} \leq S$ such that $\psi(1)_{p}=\left|S: Q_{i}\right|$ for $i=1, \ldots, n$. By Sylow's theorem, there exist representatives $g_{1}, \ldots, g_{k} \in G$ for the double cosets $H \backslash G / P$ such that $S_{i}:=H^{g_{i}} \cap P \leq S^{g_{i}}$ for $i=1, \ldots, k$. The Mackey formula (see [13, Theorem 17.4]) yields

$$
\chi_{P}=\left(\psi^{G}\right)_{P}=\sum_{i=1}^{k}\left(\left(\psi^{g_{i}}\right)_{S_{i}}\right)^{P}=\sum_{i=1}^{k}\left(\left(\left(\psi_{S}\right)^{g_{i}}\right)_{S_{i}}\right)^{P}=\sum_{i=1}^{k} \sum_{j=1}^{n}\left(\left(\left(\lambda_{j}^{g_{i}}\right)^{S^{g_{i}}}\right)_{S_{i}}\right)^{P} .
$$

Here, $\left(\left(\lambda_{j}^{g_{i}}\right)^{S^{g_{i}}}\right)_{S_{i}}$ is a sum of characters induced from $Q_{j}^{g_{i} x} \cap S_{i}$ for some $x \in S^{g_{i}}$. Since

$$
\chi(1)_{p}=|G: H|_{p} \psi(1)_{p}=\left|G: Q_{j}\right|_{p} \leq\left|P: Q_{j}^{g_{i} x} \cap S_{i}\right|,
$$

we can find $Q_{j}^{g_{i} x} \cap S_{i} \leq T_{i j}^{(x)} \leq P$ such that $\chi(1)_{p}=\left|P: T_{i j}^{(x)}\right|$. Hence, the claim holds for $\chi$.

By using Lemma 6 and Isaacs' theory of $\pi$-special characters, we can now prove Theorem 1

Proof of Theorem 1. We proceed by induction on the order of $G$. By Lemma 6, we can assume $\chi$ to be primitive. By a theorem of Isaacs (see [13, Theorem 40.8]), $\psi=\alpha \beta$ where $\alpha$ is $p$-special and $\beta$ is $p^{\prime}$-special. Thus, $\chi(1)_{p}=\alpha(1)$. By a theorem of Gajendragadkar (see [13, Theorem 40.11]), we deduce that $\alpha_{P} \in \operatorname{Irr}(P)$. Since $P$ is an M-group, there exists $T \leq P$ and a linear character $\mu \in \operatorname{Irr}(T)$ such that $\mu^{P}=\alpha_{P}$. It follows that

$$
\chi_{P}=\alpha_{P} \beta_{P}=\mu^{P} \beta_{P}=\left(\mu \beta_{T}\right)^{P},
$$

where the last equality follows by [13, Theorem 17.3]. Since $\chi(1)_{p}=\alpha(1)=|P: T|$ the result follows.

Given the proof above, it is natural to ask whether we can replace $p$ by an arbitrary set of primes $\pi$ and $p$-solvable groups by $\pi$-separable groups. The only additional hypotheses we require is that every $\pi$-subgroup of $G$ is an M-group and we can always find the groups $T_{i j}^{(x)}$ in the proof of Lemma 6 , Both requirements are true whenever $G$ has nilpotent $\pi$-Hall subgroups.

Next, we obtain Corollary 2 from Theorem 1 via an application of the Mackey formula.

Proof of Corollary 2. Let $P$ be a $p$-subgroup of $G$ and $\chi \in \operatorname{Irr}(G)$ with $\chi(1)_{p} \geq|G: P|_{p}$. Consider $S \in \operatorname{Syl}_{p}(G)$ such that $P \leq S$. By Theorem 1 there exist $Q_{i} \leq S$ and characters $\lambda_{i}$ of $Q_{i}$ such that $\chi(1)_{p}=\left|S: Q_{i}\right|$ and $\chi_{S}=\sum_{i} \lambda_{i}^{S}$. By the Mackey formula $\chi_{P}$ is a sum of the characters $\left(\lambda_{i}^{S}\right)_{P}=\sum\left(\left(\lambda_{i}^{x}\right)_{Q_{i}^{x} \cap P}\right)^{P}$, for some $x \in S$. For every such $x$, we find $Q_{i}^{x} \cap P \leq R_{i, x} \leq P$ such that $\chi(1)_{p}=\left|G: R_{i, x}\right|_{p}$. Set $\mu_{i, x}:=\left(\left(\lambda_{i}^{x}\right)_{Q_{i}^{x} \cap P}\right)^{R_{i, x}}$. Now $\chi_{P}$ is a sum of the characters $\mu_{i, x}^{P}$. 
Proof of Corollary 3. By Theorem 1, there exist subgroups $Q_{i} \leq P$ and characters $\lambda_{i} \in \operatorname{Irr}\left(Q_{i}\right)$ such that $\chi(1)_{p}=\left|P: Q_{i}\right|$ and $\chi_{P}=\sum_{i} \lambda_{i}^{P}$. Then $\chi(1)_{p}=\chi(1)_{p} \sum_{i} \lambda_{i}(1)$ and hence there exists $j$ such that $\lambda_{j}$ is linear. Since $\lambda_{j}^{P}$ is a summand of $\chi_{P}$ the result follows.

Proof of Corollary 4. We apply Corollary 2 with $P=D$. Let $\theta \in \operatorname{Irr}(D)$ be a linear constituent of $\chi_{D}$. Then there exist $Q \leq D$ and $\lambda \in \operatorname{Irr}(Q)$ such that $\chi(1)_{p}=|G: Q|_{p}, \theta$ occurs in $\lambda^{D}$ and $\lambda^{D}$ is a summand of $\chi_{D}$. By Frobenius reciprocity, this yields $\lambda=\theta_{Q}$ and $\left(\theta_{Q}\right)^{D}$ is a summand of $\chi_{D}$. Since $\chi$ has positive height, $\chi(1)_{p}>|G: D|_{p}$ and therefore $Q<D$. Choose $Q \leq R \leq D$ such that $|D: R|=p$. By Gallagher's theorem (see [13, Theorem 19.5]), $\left(\theta_{R}\right)^{D}$ is a sum of $p$ distinct linear characters and they all appear in $\chi_{D}$ since $\theta_{R}$ is a constituent of $\left(\theta_{Q}\right)^{R}$.

\section{Evidence for Conjecture 5}

In this section we collect some evidence for Conjecture 5. We start with an analysis of minimal counterexamples.

Theorem 7. Let $G$ be a minimal counterexample to Conjecture 5 subject to $(|G: \mathrm{Z}(G)|,|G|)$. Then the following holds:

(a) $G=\mathrm{O}^{p^{\prime}}(G)$.

(b) $G$ is not a direct product of proper subgroups.

(c) $\chi$ is primitive.

(d) $\chi$ is faithful.

(e) Every abelian normal subgroup of $G$ is cyclic and central.

(f) $\mathrm{O}_{p^{\prime}}(G) \leq \mathrm{Z}(G) \cap G^{\prime}$.

Proof. First note that Conjecture 5 holds for all proper subgroups $H<G$ and all proper quotients $G / N$ since

$$
\begin{aligned}
|H: \mathrm{Z}(H)| & \leq|H: H \cap \mathrm{Z}(G)|=|H \mathrm{Z}(G): \mathrm{Z}(G)| \leq|G: \mathrm{Z}(G)|, \\
|G / N: \mathrm{Z}(G / N)| & \leq|G: \mathrm{Z}(G) N| \leq|G: \mathrm{Z}(G)| .
\end{aligned}
$$

(a) Suppose that $N:=\mathrm{O}^{p^{\prime}}(G)<G$. Then $P \leq N$. By Clifford theory, $\chi_{N}$ is a sum of characters $\psi \in \operatorname{Irr}(N)$ such that $\chi(1)_{p}=\psi(1)_{p}$. Since Conjecture 5 holds for $\psi$, it must also hold for $\chi$ contradicting the choice of $G$. Consequently, $N=G$.

(b) Suppose that $G=G_{1} \times G_{2}$ for proper subgroups $G_{1}$ and $G_{2}$. Let $P=P_{1} \times P_{2}$ and $\chi=\chi_{1} \times \chi_{2}$ with $P_{i} \in \operatorname{Syl}_{p}\left(G_{i}\right)$ and $\chi_{i} \in \operatorname{Irr}\left(G_{i}\right)$ for $i=1,2$. By hypothesis, we can write $\left(\chi_{i}\right)_{P_{i}}=\sum_{k=1}^{n_{i}} a_{i k} \lambda_{i k}^{P_{i}}$ with $a_{i k} \in \mathbb{Z}$ and $\lambda_{i k} \in \operatorname{Irr}\left(Q_{i k}\right)$ where $Q_{i k} \leq P_{i}$ and $\chi_{i}(1)_{p}=\left|P_{i}: Q_{i k}\right|$. Then

$$
\chi_{P}=\left(\chi_{1}\right)_{P_{1}} \times\left(\chi_{2}\right)_{P_{2}}=\sum_{k=1}^{n_{1}} \sum_{l=1}^{n_{2}} a_{1 k} \lambda_{1 k}^{P_{1}} \times a_{2 l} \lambda_{2 l}^{P_{2}}=\sum_{k, l} a_{1 k} a_{2 l}\left(\lambda_{1 k} \times \lambda_{2 l}\right)^{P}
$$

and $\chi(1)_{p}=\chi_{1}(1)_{p} \chi_{2}(1)_{p}=\left|P_{1}: Q_{1 k}\right|\left|P_{2}: Q_{2 l}\right|=\left|P: Q_{1 k} \times Q_{2 l}\right|_{p}$ for all $k, l$. This means that Conjecture 5 holds for $G$, a contradiction. Consequently, $G$ is not a direct product of proper subgroups. 
(c) This can be shown as in Lemma 6.

(d) Suppose that $N:=\operatorname{Ker}(\chi) \neq 1$. We may regard $\chi$ as a character of $\bar{G}:=G / N$. Since $\bar{P}:=P N / N$ is a Sylow $p$-subgroup of $\bar{G}$, there exist $\overline{Q_{i}} \leq \bar{P}$ and $\lambda_{i} \in \operatorname{Irr}\left(\overline{Q_{i}}\right)$ such that $\chi_{\bar{P}}=\sum_{i=1}^{n} a_{i} \lambda_{i}^{\bar{P}}$ for some $a_{i} \in \mathbb{Z}$. Choose $Q_{i} \leq P$ such that $\overline{Q_{i}}=Q_{i} N / N$ and $Q_{i}=Q_{i} N \cap P$ for $i=1, \ldots, n$. Note that $P \cap N=Q_{i} \cap N$. Using the canonical isomorphism $Q_{i} / Q_{i} \cap N \rightarrow \overline{Q_{i}}$, we can identify $\lambda_{i}$ with the corresponding character of $Q_{i}$. This allow us to write $\chi_{P}=\sum_{i} a_{i} \lambda_{i}^{P}$. Since $\left|Q_{i} N: Q_{i}\right|=\mid Q_{i} N$ : $Q_{i} N \cap P|=| P N: P \mid, Q_{i}$ is a Sylow $p$-subgroup of $Q_{i} N$. It follows that

$$
\chi(1)_{p}=\left|\bar{G}: \overline{Q_{i}}\right|_{p}=\left|G: Q_{i} N\right|_{p}=\left|P: Q_{i}\right| .
$$

Hence, the claim would hold for $G$. Consequently, $\chi$ must be faithful.

(e) Let $N \unlhd G$ be abelian. By (ㄷ),$\chi_{N}=\chi(1) \theta$ for some linear $\theta \in \operatorname{Irr}(N)$. By (d),$\chi$ is faithful and so must be $\theta$. Hence, $N \cong \theta(N) \leq \mathbb{C}^{\times}$is cyclic. As $\theta$ is $G$-invariant, we obtain $N \leq \mathrm{Z}(G)$.

(f) By (回) we have $N:=\mathrm{O}_{p^{\prime}}(G) \leq G^{\prime}$. By (드), there is a unique $\theta \in \operatorname{Irr}(N)$ under $\chi$. Suppose that $N \nsubseteq$ $\mathrm{Z}(G)$. By [16, Problem (6.3)], there exists a character triple isomorphism $(G, N, \theta) \cong\left(G^{*}, N^{*}, \theta^{*}\right)$ such that $N^{*}$ is a central $p^{\prime}$-subgroup of $G^{*}$ and $\theta^{*}$ is linear. Since $\mathrm{O}_{p^{\prime}}\left(G^{*} / N^{*}\right) \cong \mathrm{O}_{p^{\prime}}(G / N)=1$, we have $N^{*}=\mathrm{O}_{p^{\prime}}\left(G^{*}\right)$. It is easy to see that $\mathrm{Z}\left(G^{*} / N^{*}\right)=\mathrm{Z}\left(G^{*}\right) / N^{*}$. In particular,

$$
\left|G^{*}: \mathrm{Z}\left(G^{*}\right)\right|=|G / N: \mathrm{Z}(G / N)| \leq|G: \mathrm{Z}(G) N|<|G: \mathrm{Z}(G)|,
$$

and so the claim holds for $\chi^{*}$. Also notice that $\chi(1)=\chi^{*}(1) \theta(1)$ and $\chi(1)_{p}=\chi^{*}(1)_{p}$ (see [16, p. 87]). Let $P^{*}$ be a Sylow $p$-subgroup of $G^{*}$ such that $(P N / N)^{*}=P^{*} N / N$. Then $\left(\chi^{*}\right)_{P^{*}}=\sum_{i} a_{i} \mu_{i}^{P^{*}}$ with $\mu_{i} \in \operatorname{Irr}\left(Q_{i}^{*}\right), Q_{i}^{*} \leq P^{*}$ and $\chi^{*}(1)_{p}=\left|G^{*}: Q_{i}^{*}\right|_{p}$. Choose $Q_{i} \leq P$ and $\lambda_{i} \in \operatorname{Irr}\left(Q_{i} N \mid \theta\right)$ such that $\left(Q_{i} N / N\right)^{*}=Q_{i}^{*} N^{*} / N^{*}$ and $\lambda_{i}^{*}=\mu_{i} \times \theta^{*}$. Now by [16, p. 87],

$$
\begin{aligned}
\left(\chi_{P N}\right)^{*} & =\left(\chi^{*}\right)_{P^{*} N^{*}}=\left(\chi^{*}\right)_{P^{*}} \times \theta^{*}=\sum_{i} a_{i} \mu_{i}^{P^{*}} \times \theta^{*} \\
& =\sum_{i} a_{i}\left(\mu_{i} \times \theta^{*}\right)^{P^{*} N^{*}}=\sum_{i} a_{i}\left(\lambda_{i}^{*}\right)^{P^{*} N^{*}}=\sum_{i}\left(a_{i} \lambda_{i}^{P N}\right)^{*} .
\end{aligned}
$$

Since $\psi \mapsto \psi^{*}$ is a bijection between $\mathbb{Z} \operatorname{Irr}(P N \mid \theta)$ and $\mathbb{Z} \operatorname{Irr}\left(P^{*} N^{*} \mid \theta^{*}\right)$, we obtain

$$
\chi_{P}=\left(\chi_{P N}\right)_{P}=\sum_{i}\left(a_{i} \lambda_{i}^{P N}\right)_{P}=\sum_{i} a_{i}\left(\lambda_{Q}\right)^{P} .
$$

Moreover, $\chi(1)_{p}=\chi^{*}(1)_{p}=\left|G^{*}: Q^{*}\right|_{p}=|G: Q|_{p}$. This contradiction finally shows that $N$ is central.

Our next goal, as mentioned in the introduction, is to prove the statement of Theorem 1 for the simple groups $\operatorname{PSL}(2, q)$ and $\mathrm{Sz}(q)$. We refer to this statement as the strong form of Conjecture 5 (we remind the reader that there are counterexamples to this stronger claim). The advantage of working with the strong form of the conjecture is that the claim carries over to quotients.

Proposition 8. Let $N \unlhd G$. If the strong form of Conjecture 5 holds for $G$, then the same is true for $G / N$.

Proof. We use the bar convention $\bar{H}:=H N / N$ for $H \leq G$. Let $P$ be a Sylow $p$-subgroup of $G$, so that $\bar{P}$ is a Sylow $p$-subgroup of $\bar{G}$. We identify the characters $\chi \in \operatorname{Irr}(\bar{G})$ with their inflation to $G$. 
By hypothesis, $\chi_{P}$ is a sum of characters $\lambda^{P}$ where $\lambda \in \operatorname{Irr}(Q)$ and $Q \leq P$ such that $\chi(1)_{p}=|G: Q|_{p}$. Since

$$
P \cap N \leq \operatorname{Ker}\left(\chi_{P}\right) \leq \operatorname{Ker}\left(\lambda^{P}\right)=\bigcap_{x \in P} \operatorname{Ker}(\lambda)^{x} \leq \operatorname{Ker}(\lambda),
$$

we can consider $\lambda$ as a character of $\bar{Q} \cong Q / Q \cap N$. Moreover $P \cap N=Q \cap N$. For $x \in P$ we compute

$$
\begin{aligned}
\lambda^{\bar{P}}(x N) & =\frac{1}{|\bar{Q}|} \sum_{\substack{y N \in \bar{P} \\
x^{y} N \in \bar{Q}}} \lambda\left(x^{y} N\right)=\frac{|Q \cap N|}{|Q|} \sum_{\substack{y(P \cap N) \in P / P \cap N \\
x^{y} \in Q}} \lambda\left(x^{y}\right) \\
& =\frac{|Q \cap N|}{|Q||P \cap N|} \sum_{\substack{y \in P \\
x^{y} \in Q}} \lambda\left(x^{y}\right)=\frac{1}{|Q|} \sum_{\substack{y \in P \\
x^{y} \in Q}} \lambda\left(x^{y}\right)=\lambda^{P}(x) .
\end{aligned}
$$

Hence, $\chi_{\bar{P}}$ is the sum of the induced characters $\lambda^{\bar{P}}$. Since $Q \cap N=P \cap N$ is a Sylow $p$-subgroup of $N$, we also obtain $\chi(1)_{p}=|G: Q|_{p}=|G: Q N|_{p}=|\bar{G}: \bar{Q}|_{p}$.

In the introduction we explained how Conjecture 5 follows from Kessar-Malle [14] when $P$ is abelian. If $P$ is cyclic, the strong form of Conjecture 5 can be shown without using the classification of finite simple groups.

Proposition 9. If $P$ is cyclic, then the strong form of Conjecture 5 holds for $G$.

Proof. Let $B$ be the $p$-block of $G$ containing $\chi$. Let $D \leq P$ be a defect group of $B$. Since the elements of $P \backslash D$ are not conjugate to elements of $D, \chi_{P}$ vanishes outside $D$ by [15, Corollary 5.9]. By the definition of character induction, it follows that

$$
\chi_{P}=\frac{1}{|P: D|}\left(\chi_{D}\right)^{P}
$$

Let $\lambda \in \operatorname{Irr}(D)$ be a constituent of $\chi_{D}$. Then $\lambda$ extends to a (linear) character $\hat{\lambda} \in \operatorname{Irr}(P)$. By Frobenius reciprocity,

$$
\left[\lambda, \chi_{D}\right]=\left[\hat{\lambda},\left(\chi_{D}\right)^{P}\right]=|P: D|\left[\hat{\lambda}, \chi_{P}\right]
$$

is divisible by $|P: D|$. Hence, $\frac{1}{|P: D|} \chi_{D}$ is a proper character of $D$. Since $\chi$ has height zero in $B$ (this was known to Brauer [2, 6C] and does not require [14]), we have $\chi(1)_{p}=|G: D|_{p}$.

We can now prove the claimed result for the groups $\operatorname{PSL}(2, q)$ and $\operatorname{Sz}(q)$.

Proposition 10. The strong form of Conjecture 5 holds for $G=\operatorname{SL}(2, q), \operatorname{PSL}(2, q)$ and $\operatorname{Sz}\left(2^{2 n+1}\right)$, where $q$ is a prime power and $n \geq 1$.

Proof. By Proposition 8 it is enough to consider $G=\mathrm{SL}(2, q)$ and $\mathrm{Sz}\left(2^{2 n+1}\right)$. We start by considering $G=\operatorname{SL}(2, q)$. If $2<p \nmid q$, then $G$ has cyclic Sylow $p$-subgroups and the claim follows from Proposition 9 , Let $p \mid q$. The Steinberg character $\chi \in \operatorname{Irr}(G)$ has $p$-defect zero and fulfills the claim with $Q=1$ by Brauer-Nesbitt's theorem mentioned in the introduction. Every other character $\chi \in \operatorname{Irr}(G)$ has $p^{\prime}$ degree and fulfills the claim for trivial reasons. Finally, let $p=2 \nmid q$. The Sylow 2-subgroup

$$
P=\left\langle x, y \mid x^{2^{n}}=y^{4}=1, x^{y}=x^{-1}\right\rangle
$$


is a quaternion group of order $2^{n+1}=\left(q^{2}-1\right)_{2}$ (recall that $\left.|G|=q^{3}-q\right)$. Let $X:=\langle x\rangle$ and $\lambda \in \operatorname{Irr}(X)$ be faithful. Then $\operatorname{Irr}(P)$ consists of four linear characters and the induced characters $\left(\lambda^{k}\right)^{P}$ of degree 2 for $k=1, \ldots, 2^{n-1}-1$. The character table of $G$ depends on $q \equiv \pm 1(\bmod 4)$ (equivalently $q \equiv \pm 1$ $\left.\left(\bmod 2^{n}\right)\right)$. It suffices to consider $\chi \in \operatorname{Irr}(G)$ with even degree. Since $y$ is conjugate to $x^{2^{n-2}}$ in $G$, the restrictions $\chi_{P}$ are determined by the following values:

\begin{tabular}{c|ccc} 
& 1 & $z$ & $x^{j}$ \\
\hline$\chi_{k}$ & $q \pm 1$ & $(-1)^{k}(q \pm 1)$ & $\pm\left(\lambda^{k}\right)^{P}\left(x^{j}\right)$ \\
$\psi_{1}$ & $q \mp 1$ & $-(q \mp 1)$ & 0 \\
$\psi_{2}$ & $\frac{q \mp 1}{2}$ & $-\frac{q \mp 1}{2}$ & 0
\end{tabular}

where $z=x^{2^{n-1}}$ and $1 \leq j, k \leq 2^{n-1}-1$. Let $Z:=\langle z\rangle=\mathrm{Z}(P)=\mathrm{Z}(G)$ and $W:=\left\langle x^{2^{n-2}}\right\rangle \cong C_{4}$. Then $\psi_{1}=\frac{q \mp 1}{2^{n}}\left(\lambda_{Z}\right)^{P}$ and $\psi_{2}=\frac{q \mp 1}{2^{n}}\left(\lambda_{W}\right)^{P}$ fulfill the claim since $\psi_{1}(1)_{2}=(q \mp 1)_{2}=2^{n}=|P: Z|$ and $\psi_{2}(1)_{2}=2^{n-1}=|P: W|$. Now suppose that $k$ is odd, so that $\left(\chi_{k}\right)_{Z}=(q \pm 1) \lambda_{Z}$. Then $\chi_{k}$ has no linear constituents, since those lie over $1_{Z}$. Hence, $\chi_{k}$ is a sum of characters induced from subgroups of index $2=(q \pm 1)_{2}=\chi_{k}(1)_{2}$.

The case $k \equiv 0(\bmod 2)$ is more complicated. Here $\chi_{k}(y)= \pm(-1)^{k / 2} 2=:(-1)^{s} 2$. Let $Y_{1}:=\left\langle x^{2}, y\right\rangle \cong$ $Q_{2^{n}}, Y_{2}:=\left\langle x^{2}, x y\right\rangle \cong Q_{2^{n}}$ and $\mu_{i} \in \operatorname{Irr}\left(Y_{i}\right)$ such that $\mu_{i}\left(x^{2}\right)=1$ and $\mu_{1}(y)=\mu_{2}(x y)=-1$. We compute

$$
\chi_{k}=\frac{q \mp 1}{2^{n}}\left(1_{Z}\right)^{P} \pm\left(\lambda^{k}\right)^{P}+\left(\mu_{1}^{s}\right)^{P}+\left(\mu_{2}^{s}\right)^{P}-\left(1_{P^{\prime}}\right)^{P},
$$

where we recall that $P^{\prime}=\left\langle x^{2}\right\rangle$. Since $k$ is even, $\lambda^{k}$ lies over $1_{Z}$ and so does $1_{P^{\prime}}$. On the other hand, $\lambda^{k}$ does not lie over $1_{P^{\prime}}$ since $k<2^{n-1}$. It follows that $\left(1_{Z}\right)^{X} \pm \lambda^{k}-1_{P^{\prime}}^{X}$ is a proper character of $X$ and $\chi_{k}$ is a sum of characters induced from $X, Y_{1}$ and $Y_{2}$. All have index $2=\chi_{k}(1)_{2}$ in $P$.

Now let $G=\operatorname{Sz}(q)$ where $q=2^{2 n+1}$ and $n \geq 1$. The Sylow $p$-subgroups for $p>2$ are cyclic by [18, Theorem 9]. Thus, again we restrict to $p=2$. The Sylow 2-subgroup $P$ is a so-called Suzuki 2-group of order $q^{2}$ such that $Z:=\mathrm{Z}(P)=P^{\prime}=\Phi(P)$ is elementary abelian of order $q$ and contains all involutions of $P$ (see [18, Theorem 7]). The character table of $G$ is given in [18, Theorem 13]. It can be seen that there are only two characters $\chi, \bar{\chi} \in \operatorname{Irr}(G)$ of even degree and not of 2-defect 0 . The values on $P$ are $\chi(1)=2^{n}(q-1), \chi(z)=-2^{n}$ and $\chi(x)=2^{n} \sqrt{-1}$ where $z \in Z \backslash\{1\}$ and $x \in P \backslash Z$. This implies $\chi_{Z}=2^{n}\left(\rho_{Z}-1_{Z}\right)$ where $\rho_{Z}$ is the regular character of $Z$. Therefore, $\chi_{P}$ has no linear constituents, because those must lie over $1_{P^{\prime}}=1_{Z}$. On the other hand, it has been shown in [17] that all non-linear characters of $P$ have degree $2^{n}$. Hence, $\chi_{P}$ is a sum of characters induced from subgroups of index $2^{n}=\chi(1)_{2}$.

As a final result we verify the strong form of Conjecture 5 if $P$ is a dihedral or quaternion 2-group. Notice that the proof of Theorem 7 applies verbatim to the strong form. This remark will be used in the following.

Proposition 11. Let $P$ be a Sylow p-subgroup of $G$ and suppose that $P$ is a dihedral or quaternion 2-group including the Klein four-group. Then the strong form of Conjecture 5 holds for $G$.

Proof. Let $G$ be a finite group with dihedral or quaternion Sylow 2-subgroup P. By Theorem 1, we may assume that $G$ is non-solvable. We argue by induction on $(|G: \mathrm{Z}(G)|,|G|)$. Since every subgroup and every quotient of $P$ is a cyclic, dihedral or quaternion group, we may apply the reduction methods from Theorem 7 (the character triple isomorphism in Theorem 7(I) preserves $P$ ). Specifically, we assume that $\mathrm{O}_{2^{\prime}}(G) \leq \mathrm{Z}(G) \cap G^{\prime}, G=\mathrm{O}^{2^{\prime}}(G)$ and $\chi$ is primitive (but not necessarily faithful). Now the GorensteinWalter theorem shows that $G$ is a Schur cover of $A_{7}, \operatorname{PSL}(2, q)$ or of $\operatorname{PGL}(2, q)$ where $q>3$ is an odd 
prime power (see [19, Theorems 6.8.7 and 6.8.9]). The first case and the exceptional cover 3. PSL $(2,9)=$ $3 . A_{7}$ can be checked by computer while $\operatorname{PSL}(2, q)$ has been considered in Proposition 10, It remains to prove the claim for $\operatorname{PGL}(2, q)$. Thanks to Proposition 8 it suffices to consider $G=\mathrm{GL}(2, q)$. The reader can find the following well-known facts in [5, §5.2], for instance.

Case 1: $G=\mathrm{GL}(2, q)$ with $q \equiv-1(\bmod 4)$.

Here $P=\left\langle x, y \mid x^{2^{n}}=y^{2}=1, x^{y}=x^{2^{n-1}-1}\right\rangle$ is a semidihedral group of order $2^{n+1}$ where $2^{n}=$ $\left(q^{2}-1\right)_{2}$. Let $z:=x^{2^{n-1}}$ be the central involution in $G$. All non-central involutions are conjugate to $y$ and all elements of order 4 are conjugate to $x^{2^{n-2}}$ in $G$. The characters of degree $q+1$ are induced from proper subgroups. There remains only one family of characters to consider. Let $X:=\langle x\rangle$ and $\lambda \in \operatorname{Irr}(X)$ be faithful. The restrictions $\chi_{P}$ assume the following values

\begin{tabular}{c|cccc} 
& 1 & $z$ & $x^{j}$ & $y$ \\
\hline$\chi_{k}$ & $q-1$ & $(-1)^{k}(q-1)$ & $-\left(\lambda^{k}\right)^{P}\left(x^{j}\right)$ & 0
\end{tabular}

where $k \not \equiv 0\left(\bmod 2^{n-1}\right)$. If $k$ is odd, then $\chi_{k}$ does not have linear constituents and the claim follows since $\chi_{k}(1)_{2}=(q-1)_{2}=2$. If on the other hand $k \equiv 0(\bmod 2)$, then

$$
\chi_{k}=\frac{q+1}{2^{n-1}}\left(\lambda_{W}\right)^{P}-\lambda^{P}=\left(\frac{q+1}{2^{n-1}}\left(\lambda_{W}\right)^{X}-\lambda\right)^{P}
$$

where $W:=\left\langle x^{2^{n-2}}\right\rangle \cong C_{4}$. The claim follows as before.

Case 2: $G=\operatorname{GL}(2, q)$ with $q \equiv 1(\bmod 4)$.

In this case

$$
P=\left\langle x, y, z \mid x^{2^{n}}=y^{2^{n}}=z^{2}=[x, y]=1, x^{z}=y\right\rangle \cong C_{2^{n}}\left\langle C_{2},\right.
$$

where $2^{n}=(q-1)_{2}$. This group can be realized conveniently by $x=\operatorname{diag}(\zeta, 1), y=\operatorname{diag}(1, \zeta)$ and $z=\left(\begin{array}{ll}0 & 1 \\ 1 & 0\end{array}\right)$ where $\zeta \in \mathbb{F}_{q}^{\times}$has order $2^{n}$. Note that $x y \in \mathrm{Z}(P) \leq \mathrm{Z}(G)$ and $z$ is conjugate to $x^{2^{n-1}}$ in $G$. Moreover, $x z$ has order $2^{n+1}$ and is conjugate to $(x z)^{2^{n}+1}=-x z$. Also, $P / Z \cong D_{2^{n+1}}$. Let $\lambda \in \operatorname{Irr}(\langle x z\rangle)$ be faithful. The values of $\chi_{P}$ are

$$
\begin{array}{c|cccc} 
& 1 & (x y)^{i} & x^{i} y^{j} & (x z)^{k} \\
\hline \chi_{l} & q-1 & (q-1) \lambda^{l}(x y)^{i} & 0 & -\left(\lambda^{l}\right)^{P}\left((x z)^{k}\right)
\end{array}
$$

where $i \not \equiv j\left(\bmod 2^{n}\right), k \equiv 1(\bmod 2)$ and $l \not \equiv 0\left(\bmod 2^{n-1}\right)$. If $l$ is odd, then $\chi_{l}$ vanishes on $(x z)^{k}$. Thus, $\chi_{l}=\frac{q-1}{2^{n}}\left(\lambda^{l}\right)^{P}$ and $\chi_{l}(1)_{2}=(q-1)_{2}=2^{n}=|P:\langle x z\rangle|$. If $l$ is even, then $\chi_{l}(x z)=-2 \lambda^{l}(x z)=$ $\left(\lambda^{l+2^{n}}\right)^{P}(x z)$ and

$$
\chi_{l}=\frac{q-1-2^{n}}{2^{n+1}}\left(\lambda^{l}\right)^{P}+\frac{q-1+2^{n}}{2^{n+1}}\left(\lambda^{l+2^{n}}\right)^{P} .
$$

By analyzing the Ree groups $G={ }^{2} G_{2}(q)$ and making use of Walter's theorem, the strong form of Conjecture 5 can be shown for all groups with abelian Sylow 2-subgroups. Since Conjecture 5 holds for abelian $P$, we omit the details.

Using GAP [6], 4ti2 [1] and its GAP interface [11], we checked the strong form of Conjecture 5 for all groups of order at most 2000. Moreover, none of the perfect groups of order at most $10^{6}$ are minimal counterexamples in the sense of Theorem 7. Additionally, the stated form of Conjecture 5 has been verified for all simple groups up to $\operatorname{PSL}(3,13)$ and all sporadic groups up to $\mathrm{Co}_{3}$ (with respect to the group order). 


\section{Acknowledgment}

We thank Gabriel Navarro for sharing his insights on a previous version of this paper, and for requesting more computer checking. We appreciate Eugenio Giannelli's effort to prove corresponding results for symmetric groups. Moreover, Alexander Hulpke has kindly provided an updated database [12] of all perfect groups of order at most $10^{6}$. Thomas Breuer has introduced the authors to numerous tricks regarding character tables in GAP. The first author is supported by the research training group GRK2240: Algebro-geometric Methods in Algebra, Arithmetic and Topology of the German Research Foundation. The second author is supported by the German Research Foundation (SA 2864/1-2 and SA 2864/3-1).

\section{References}

[1] team4ti2, 4ti2-A software package for algebraic, geometric and combinatorial problems on linear spaces, https://4ti2.github.io.

[2] R. Brauer, On blocks and sections in finite groups. II, Amer. J. Math. 90 (1968), 895-925.

[3] R. Brauer and C. Nesbitt, On the modular characters of groups, Ann. of Math. (2) 42 (1941), $556-590$.

[4] E. Cline, On minimal vertices and the degrees of irreducible characters, J. Algebra 24 (1973), 379-385.

[5] W. Fulton and J. Harris, Representation theory, Graduate Texts in Mathematics, Vol. 129, Springer-Verlag, New York, 1991.

[6] The GAP Group, GAP - Groups, Algorithms, and Programming, Version 4.11.0; 2020, (http://www.gap-system.org).

[7] E. Giannelli, On the restriction of irreducible characters of symmetric groups to Sylow p-subgroups, J. Algebra 483 (2017), 37-57.

[8] E. Giannelli, A note on restriction of characters of alternating groups to Sylow subgroups, J. Algebra 521 (2019), 200-212.

[9] E. Giannelli and G. Navarro, Restricting irreducible characters to Sylow p-subgroups, Proc. Amer. Math. Soc. 146 (2018), 1963-1976.

[10] E. Giannelli and B. Sambale, On restriction of characters to defect groups, J. Algebra 558 (2020), 423-433.

[11] S. Gutsche, GAP Package 4ti2Interface, https://homalg-project.github.io/homalg_project/4ti2Interf

[12] A. Hulpke, The perfect groups of order up to two million, arXiv:2104.10828v3.

[13] B. Huppert, Character theory of finite groups, De Gruyter Expositions in Mathematics, Vol. 25, Walter de Gruyter \& Co., Berlin, 1998.

[14] R. Kessar and G. Malle, Quasi-isolated blocks and Brauer's height zero conjecture, Ann. of Math. (2) 178 (2013), 321-384.

[15] G. Navarro, Characters and blocks of finite groups, London Mathematical Society Lecture Note Series, Vol. 250, Cambridge University Press, Cambridge, 1998. 
[16] G. Navarro, Character theory and the McKay conjecture, Cambridge Studies in Advanced Mathematics, Vol. 175, Cambridge University Press, Cambridge, 2018.

[17] I. A. Sagirov, Degrees of irreducible characters of Suzuki 2-groups, Mat. Zametki 66 (1999), 258263 (translation in Math. Notes 66 (1999), 203-207).

[18] M. Suzuki, On a class of doubly transitive groups, Ann. of Math. (2) 75 (1962), 105-145.

[19] M. Suzuki, Group theory. II, Grundlehren der Mathematischen Wissenschaften, Vol. 248, SpringerVerlag, New York, 1986.

[20] T. Wilde, Orders of elements and zeros and heights of characters in a finite group, arXiv:math/0604337v1.

[21] W. Willems, A note on Brauer's induction theorem, J. Algebra 58 (1979), 523-526. 